\title{
AgénésiePartielle Du Septum Pellucidum Isolée : Un Diagnostic Anténatal
}

\author{
Kabulukadingi Arnauld ${ }^{1,2 *}$, AmaniMaleya Chuma ${ }^{1}$, Kabila Mfyama \\ Marcel $^{1}$, MwilambweNgoy Steve ${ }^{1}$, PatauleFatuma Honorine ${ }^{1}$, Pierre Bernard ${ }^{2}$. \\ ${ }^{1}$ Département De Gynécologie et Obstétrique, Faculté De Médecine/Université De Lubumbashi, RD Congo. \\ ${ }^{2}$ Service d'Obstétrique des cliniques universitaires Saint Luc de Bruxelles, Université Catholique de Louvain, \\ Belgique.
}

\begin{abstract}
The septum pellicidumis a thin membrane locatedat the midline of the brainbetween the twocerebralhemispheres, or halves of the brain. It isconnected to the corpus callosum(collection of nerve fibersthatconnect the cerebralhemispheres), the septum pellicidumagenesiais a rare abnomalityaccompagniesvarious malformations of the brainthat affect intelligence, behavoir, and the neurodevelopmentalprocess. We report, in thisarcticle, a case ofisolated partial absence of the septum pellucidum in a fetus of 28 weeks and twodays. The diagnosis has been done by an antenatalmorphologicalultrasound scan and MRI. Wewant to emphasize the importance of visualisation of the cavum of the septum pellucidum as one of the items to notice whileperformingmorphologic scan of the fetalbrain.
\end{abstract}

Keywords : Partial agenesia, Septum pellicidum, antenatal.

\section{Introduction}

Le septum pellucidum (SP) est une structure membraneuse de la ligne médiane, constituée de deux feuillets et qui sépare les ventricules latéraux. Dans sa partie supérieure en antérieur, il est attaché au corps calleux et dans sa partie inferieure en postérieur il est attaché à la face supérieure du fornix.

L'agénésie partielle ou complète du SP est rapportée dans 2 à 3 cas sur 100000 naissances dans la population générale. L'agénésie isolée du SP est très rare mais généralement elle est associée à d'autres malformations cérébrales notamment, l'holoprosencéphalie, la dysplasie septo-optique, laschizencéphalie, l'agénésie du corps calleux, ...

Nous rapportons ici un cas d'agénésie partielle du septum pellucidum à priori isolée.

\section{Observation}

Madame K.N, primigeste âgée de 23 ans, est référée à l'unité du diagnostic anténatal(DAN) du service d'obstétrique des cliniques universitaires Saint Luc de Bruxelles pour suspicion de malformation cérébrale chez le foetus qu'elle porte, probable agénésie du septum pellucidum.

Elle présentait des antécédents marqués par:Médico-chirurgicaux: une intolérance au glucose traitée par metformine stoppée à 5 semaines de grossesse et depuis sous insuline.

Gynéco-obstétriques : G1 P0 et le terme est prévu pour le 30/09/2013

Familiaux :

Cousin germain paternel âgé de 35 ans présente un retard mental sans diagnostic précis.

Oncle maternel décédé à l'âge de 9 ans et présentait un retard mental sans diagnostic précis.

Chez le père du fœetus : un frère paraplégique qui est porteur d'un spina bifida avec microcéphalie et un retard mental.

Pour ce qui est de la grossesse actuelle : la patiente est A rhésus positif, protégée contre la rubéole et la toxoplasmose mais pas contre le cytomégalovirus.Nous n'avons pas d'information sur l'échographie du premier trimestre ni sur la clarté nucale, le triple test serait normal d'après la patiente. A l'échographie morphologique les structures cérébrales sont mal visualisées et c'est lors du complément morphologique que l'on soupçonne une malformation cérébrale.

A son admission au DAN à 28 semaines $2 / 7$ gestationnelles une échographie est faite et montre ce qui suit : une biométrie parallèle au percentile 28 et 92 (PC // p83, PA // p 28, FL // p43, Poids: 1153 g // p50; VP : $8,6 \mathrm{~mm}$, Cervelet // p 60)

Au niveau des structures cérébrales : Sur la coupe frontale, les parois du cavum du septum pellucidum ne sont pas individualisées, Il y a une fusion des deux cornes frontales (Fig. A et B), ce qui conduit à une suspicion d'agénésie partielle du SP, les ventricules postérieurs de $8,6 \mathrm{~mm}$, le 3ème ventricule est d'aspect 
normal, le corps calleux est présent et normal ainsi que l'artère péricalleuse (Fig. C), les sillons calcarins ont un aspect normal, pas de colpocéphalie et le reste de la morphologie du foetus est normal.

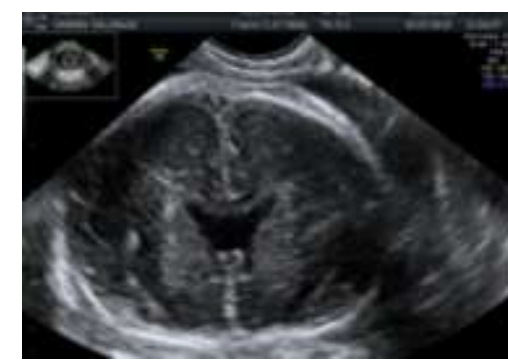

A

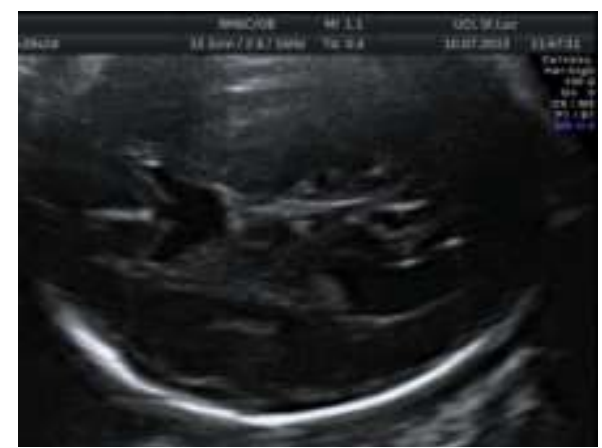

$\mathrm{D}$

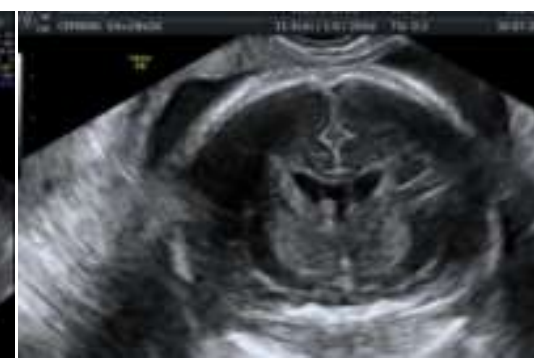

$\mathrm{B}$

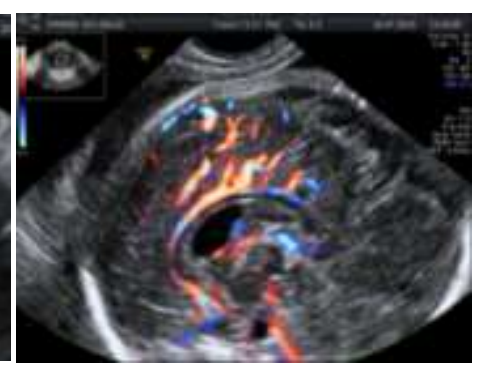

$\mathrm{C}$

Figure 1. A, B : fusion des deux cornes frontales ; C : corps calleux bien visualisé et trajet normal de l'artère péricalleuse ; D : dimension normale du ventricule postérieur ; E : la distance normale entre les deux orbites ; F : oreille bien ourlée.

Une amniocentèse a été réalisée par voie transplacentaire sans particularités, et le liquide envoyé au laboratoire pour analyses génétiques :

FISH 13,18 et 21 sont négatifs, le caryotype 46, XY

La CGH note une microdélétion interstitielle sur le chromosome $6: 6 q 15-16.1$ couvrant le gène EPHA 7, Cette anomalie est de novo car non retrouvée chez les parents.

La patiente est revue à 34 semaines $3 / 7$ gestationnelles :

- à l'échographie :

Agénésie du SP, le corps calleux bien visualisé et normal, VP 7,7 mm, pas d'hyper ni d'hypotélorisme, les angles faciaux normaux, oreilles semblent bien ourlées avec une hauteur de $30 \mathrm{~mm}(\mathrm{P} 80)$, la face et le profil sont bien visualisés et normaux, OPN 9,8 mm (P 40).

\section{- à l'IRM :}

La maturation cérébrale est normale pour l'âge gestationnel, le corps calleux et le chiasma optique présents, les nerfs optiques sont présents de deux cotés, fins et symétriques (il n'est pas possible d'affirmer l'hypoplasie des nerfs), les cavumpellucidum et cavumvergae sont d'aspects normaux; on note une disposition habituelles des ventricules latéraux (, Fig. G, H, I.) il n'y a pas d'évidence de foyer hémorragique ou d'anomalie supra ou infratantorielle.

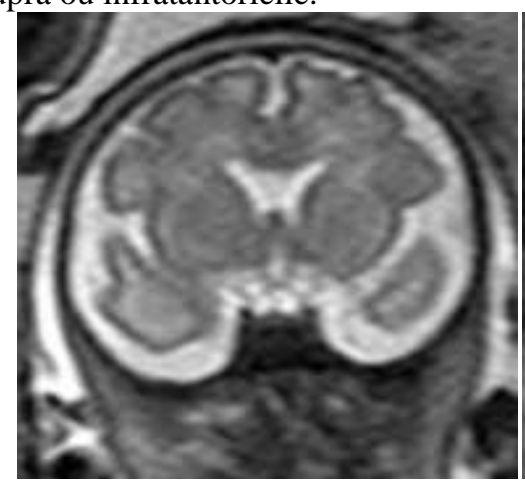

G

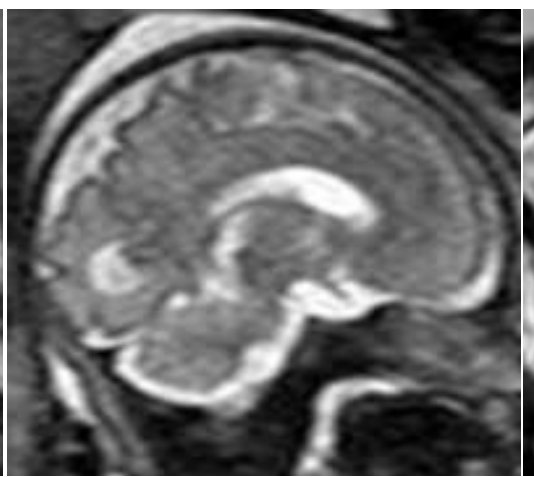

$\mathrm{H}$

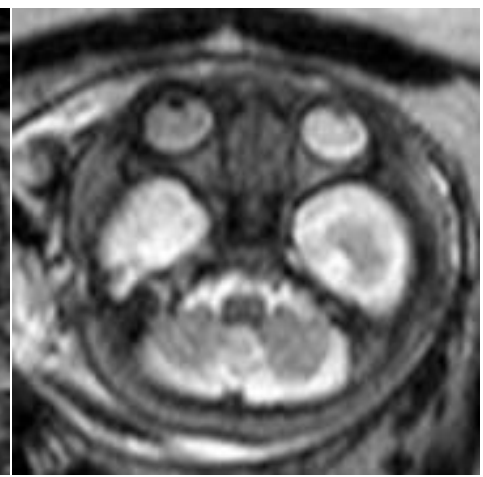

I 


\section{Avis de la Neuropédiatrie}

L'agénésie septale sans autres malformations cérébrales est de bon pronostique, mais la dysplasie septo-optique n'est pas exclue même avec l'IRM rassurante.

Les parents, après entretien avec le généticien et le neuropediatre sont désireux de poursuivre la grossesse et accoucher avec le gynécologue référant.

L'accouchement a eu lieu par voie basse à 37 SA 6/7, un garçon, 3760g, apgar 9-10-10 L'enfant en parfaite santé à 7 mois de vie.

\section{Discussion}

L'agénésie du septum pellucidum (ASP) est un defect congénital dans le développement des structures télencéphaliquesde la ligne médiane. Elle peut être primitive ou résultat d'un phénomène acquis, allusion faite à la nécrose du SP suite, notamment, à une hydrocéphalie sévère prolongée.

L'agénésie du SP est le plus souvent associée à d'autres malformations cérébrales comme, ladysplasiesepto-optique (SOD), l'agénésie du corps calleux (ACC), l'holoprosencéphalie (HPE) ou l'hydrocéphalie, ...

En Israel, dans une série de 25 foetus avec agénésie du SP, 12 avaient des images échographiques classiques suggestives d'HPE dont 8 HPE alobaire et 4 HPE sémilobaire, les autres foetus ont eu comme malformations cérébrales associées : SOD, hydrocéphalie, schizencéphalie, l'ACC, porencéphalie [1]. Il est à noter que l'agénésie pouvant être associée aux anomalies chromosomiques, sur 6 analyses du caryotype disponible dans sa série, deux foetus étaient porteurs d'une trisomie 13 et un d'une triploïdie.

En 2010 à Seoul deux cas d'agénésie du SP associé une fusion de deux parties du fornix et une ventriculomégalie ont été décrit [2].

L'agénésie du SP congénitale isolée est par contre très rare 2 à 3 cas pour 100000 naissances, un cas a été décrit en Espagne par l'équipe de Garcia-Arrez avec une confirmation du diagnostic et un follow-up post natal révélant un bon pronostic jusqu'à 18 mois [3]. Notons qu'une équipe italienne quant à elle a décrit un cas d'ASP isolée dont le diagnostic a été confirmé à l'autopsie les parents ayant décidé d'interromprela grossesse [4].

Nous avons rapporté ici un cas d'ASP à priori isolée chez un fœus porteur d'une microdélétion interstitielle sur le chromosome $6: 6 q 15-16.1$ couvrant le gène EPHA 7, d'après la littérature, ce gène joue un rôle dans le développement du système nerveux, une anomalie de novo. Diagnostic confirmé à l'IRM anténatale.

Pour ce qui est du pronostic postnatal d'ASP, il dépend du fait d'être isolée ou associée aux autres malformations cérébrales; ainsi donc il est souvent bon lorsque l'ASP est isoléemais par contre mauvais lorsqu'associée aux malformations cérébrales, notamment à une SOD, elle peut s'accompagner des troubles visuels pouvant aller jusqu'à une cécité, un panhypopituitarisme et retard mental [5].

\section{Conclusion}

Le cavum du septum pellucidum est une structure sentinelle dans le dépistage prénatal des anomalies de la ligne médiane. Son repérage systématique se justifie par la gravité de certaines malformations dans lesquelles le septum est impliqué. La visualisation du cavum constitue un des items clé à préciser lors des échographies de dépistage de 22 et de 32 semaines d'aménorrhée. Un examen de référence doublé d'une IRM foetale est à prévoir devant toute absence d'image de cavum, à la recherche d'anomalies associées (morphométrie ventriculaire, intégrité du corps calleux, des voies optiques, du cortex et de la face).

Conflits d'intérêt: Aucun.

Contribution des auteurs: Tous les auteurs ont participé à la réalisation de ce travail, ils ont lu et approuvé la version finale du manuscrit.

\section{Références}

[1]. Malinger G., Lev D., Kidron D., Heredia F., Hershkovitz R., Leman-Sagie T : differentialdiagnosis in fetuseswith absent septum pellucidum, UltrasoundobstetGynecol 2005, $25: 42-49$.

[2]. Chun Y.k., Kim H. S., Hong S. R., Chi J.G. : Absence of the septum Pellucidumassocietedwith a midlinefornical nodule and ventriculomégaly : A report of two cases, J Korean Med Sci 2010, 25 :970-3.

[3]. Garcia-Arrez A., Garcia-Diaz L., Fajardo M., Carreto P., Antinolo G :Isolated absence of septum pellucidum : Prenataldiagnosis and outcome, FetalDiagnTher 2013, 33, 130-132.

[4]. Pilu G., Tani G., Carletti A., Malaiga S., Ghi T., Rizzo N :Difficultearlysonographicdiagnosis of absence of the fetal septum pellucidum, UltrasoundobstetGynecol 2005, $25: 72-76$.

[5]. Damaj L., Bruneau B., Ferry M., Moutard, Garel C., Odent S., Adamsbaum C., Avni F., Treguier C., Lazarol : Pediatricoutcome of childrenwith the prenataldiagnosis of isolatedsetalagenesis, PrenatalDiagnosis $2010,30: 1143-50$. 